\title{
Universal Equivalent Static Wind Load Estimation for Spatial Structures based on Wind-induced Envelope Responses
}

\author{
Yuan-Qi LI *, Lei WANG ${ }^{\text {a }}$, \\ Yukio TAMURA ${ }^{\mathrm{b}}, \mathrm{Zu}^{\mathrm{T}}$ Yan $\mathrm{SHEN}^{\mathrm{a}}$ \\ * Dept. of Building Engineering, Tongji University, \\ Shanghai, 200092, P. R. of China \\ liyq@mail.tongji.edu.cn \\ ${ }^{a}$ Dept. of Building Engineering, Tongji University, Shanghai, P. R. of China \\ ${ }^{\mathrm{b}}$ Wind Engineering Research Center, Tokyo Polytechnic University, Atsugi, Japan
}

\begin{abstract}
With the rapid development of new materials and structural techniques, many new kinds of spatial structural systems have been widely used for large-span roofs, such as the sport, exhibition and entertainment halls, and they are becoming more and more large-span and flexible. However, up to now, wind-resistant design of large-span spatial structures is still complex and difficult in practice due to their strong individuality and complexity of architectural shapes and lack of practical and feasible provisions in related codes for structural design especially. In this paper, a practical wind-resistant approach based on Wind-induced Envelope Responses, i.e., the maximum or minimum responses, including displacement of any nodes, internal forces (axial force, bending moment, torsional moment, or extreme stress, etc.) of any elements, reaction of any supports, etc., of the structures in question under the expected wind load action with any attack angle, is presented for this problem from the structural designers' point of view, and the advantages and disadvantages of the existing approaches used in wind-resistant design of large-span spatial structures, as well as the presented one, are analyzed at different aspects, such as necessary information of wind load, time consuming problem, convenience in practice, and so on. Finally, the convenience and efficiency of the presented approach is illuminated with two numerical examples of single layered reticulated shells based on the pressure information obtained from wind tunnel tests.
\end{abstract}

Keywords: wind-resistant design, large-span spatial structures (LSS), equivalent static wind load (ESWL), wind-induced envelope responses (WER) 


\section{Introduction}

With the rapid development of new materials and structural techniques, many new kinds of spatial structural systems have been widely used for large-span roofs, such as the sport, exhibition and entertainment halls, and they are becoming more and more large-span and flexible [1]. For the structural design of such spatial structures, wind load may be very important since these structural systems are relatively light, and they may be sensitive to the external load distribution and the stability may be an important, even dominant, problem for those with shell shape. At present, more and more research attentions have been paid to wind-resistant design of large-span spatial structures, and important conceptions and approaches have been established for this important issue. However, it is still a complex and difficult job in practice to conduct a reliable wind-resistant design from case to case mainly due to the following reasons: (1) as a architectural symbol of a city, the envelope shape of a large-span spatial structure usually has strong individuality, and most of them are complex with curved shapes. Therefore, it is almost impossible to get reliable wind load information directly from a loading code in effect, and wind tunnel investigation is always necessary to do in order to offer some fundamental information about the wind pressure distribution on the surface of the structure; and (2) up to now, only some basic provisions are given in related codes for structural design [2,3], and wind-resistant design of large-span spatial structures can not be simply conducted by following the provisions directly. From the structural designer's point of view, how to get a simple and reliable way to consider wind-reduced responses in combination of load and action responses is an important problem for wind-resistant design of large-span spatial structures with different structural systems.

In this paper, with brief review and comparison of the existing approaches used in windresistant design of large-span spatial structures, especially for the situation in China, a practical approach based on Wind-induced Envelope Responses was presented for this problem, and the advantages and disadvantages of the above methods are analyzed at different aspects, such as necessary information of wind load, time consuming problem, convenience in practice, and so on. Finally, convenience and efficiency of the presented approach is illuminated with two numerical examples of single layered shells.

\section{Brief review on wind-resistant design methods for spatial structures}

\subsection{Equivalent static wind loading distribution}

Since wind loading has great effects on the limit load-carrying capacities, or sometimes the stability of large-span spatial structures, it is critical to estimate the equivalent static wind load for structural wind-resistant design. During the estimating process, several aspects should be considered, including the mean wind load as static effect, the fluctuating component as dynamic effect with random characteristics, and even the interaction between wind pressure and structural surface as aeroelastic effect. In the following, several existing equivalent static wind loading estimation methods were discussed and compared.

\subsubsection{The gust response factor type methods (GRF)}


In the gust factor type methods [4], the wind load vector of a structure, $\left\{F_{W}\right\}$, can be calculated with a given gust factor $G_{f}$ and the mean wind load vector, $\left\{\bar{F}_{w}\right\}$, as

$$
\left\{F_{W}\right\}=G_{f}\left\{\bar{F}_{w}\right\}
$$

Taking the estimation method recommended by the loading code of China [2] as an example, the following equation is used:

$$
w_{k}=w_{0} \mu_{z} \beta_{z} \mu_{s}
$$

where, $w_{0}$ is the basic wind pressure in site; $\mu_{\mathrm{z}}$ is the height coefficient, a modifying coefficient for the height of the point; $\mu$ s is the shape coefficient, a modifying coefficient for the place of the point on the surface; $\beta_{\mathrm{z}}$ is the wind-excited vibration coefficient.

Then, the wind load vector, $\left\{F_{W}\right\}$, can be obtained by multiplying the corresponding tributary area vector of a shell, $\{A\}$, as

$$
\left\{F_{W}\right\}=w_{0} \mu_{z} \beta_{z} \mu_{s}\{A\}=\beta_{z}\left(\mu_{z} \mu_{s} w_{0}\{A\}\right)
$$

Here, the result of $\left(\mu_{\mathrm{z}} \mu_{\mathrm{s}} \beta_{\mathrm{z}} w_{0}\{A\}\right)$ can be taken as the mean wind load vector, and the windexcited vibration coefficient. $\beta_{\mathrm{z}}$ is used as a gust factor to reflect the effects of fluctuating component. Usually, an empirical value about 1.5 2.0 is used for all the nodes of a spatial structure.

The GRF method were proposed by Davenport for the wind resistant analysis of high-rise buildings. Due to its simplicity, it was still used in the design of large span structures. In such methods, it implies that the distribution of wind force resulted from fluctuating component is as the same as that from the mean wind pressure. However, it is not the fact in many cases. The shortcomings of such equivalent static wind loading distribution estimation methods for wind-resistant design of some typical spatial structures, such as single-layer reticulated spherical shells, are obvious, although such methods are really simple and palatable for design engineers.

\subsubsection{The effective static wind load distribution estimation method (ESWL)}

The equivalent static wind loading distribution can be estimated by the effective static wind load distribution estimation to get the extreme values of a reference response of interest. The effective static wind load distribution for a structure can be separately derived for three components as follows [4]:

$$
\left\{F_{i}\right\}=\left\{\bar{F}_{i}\right\}+W_{B}\left\{F_{B i}\right\}+W_{R}\left\{F_{R i}\right\}
$$

where, $F_{i}, F_{B i}$ and $F_{R i}$ are the mean component, the background or sub-resonant component and the resonant component, respectively, at node i; $W_{B}$ and $W_{R}$ are the weighting factors given by

$$
\left|W_{B}\right|=\frac{g_{B} \sigma_{r, B}}{\left(g_{B}^{2} \sigma_{r, B}^{2}+g_{R}^{2} \sigma_{r, R}^{2}\right)^{1 / 2}}
$$




$$
\left|W_{R}\right|=\frac{g_{R} \sigma_{r, R}}{\left(g_{B}^{2} \sigma_{r, B}^{2}+g_{R}^{2} \sigma_{r, R}^{2}\right)^{1 / 2}}
$$

where $g_{B}$ and $g_{R}$ are the peak factors of background and resonant component, respectively; $\sigma r, \mathrm{~B}$ and $\sigma \mathrm{r}, \mathrm{R}$ are the standard deviations of background and resonant component of response variable of interest, $r$.

With the effective static wind load distribution estimation method, the equivalent static wind load distribution can be estimated in order to obtain a maximum or minimum value of a response variable of interest, efficiently. However, there is still a problem, i.e., how to determine a suitable reference response initially in order to get the most unfavorable wind load distribution [5], since there are so many critical response variables in a practical project which are necessary to be considered in strength, stability or deflection analysis, respectively.

\subsubsection{The most unfavorable distribution estimation method [6] (Unfavorable ESWL)}

For some typical spatial structures, such as large-span single-layer reticulated shells, the main questions in structural design may be deformation and stability problems, and the random characteristics of fluctuating wind load can also be looked as a type of loading imperfection, the corresponding possible instability mode of shell can be used to give a conservative estimation on the effects of fluctuating component of wind loading. Such a method, called "the conformable imperfection mode method", is often used in sensitivity analysis of any other imperfections, which has been proved to be very efficient. A simple method based on the conformable imperfection mode method was presented in Ref. [6]. Supposing under a load combination including the mean wind force vector $\left\{F_{W}\right\},\left[K_{\mathrm{t}}\right]$ becomes non-positive at the $i+1$-th incremental step, which means a limit or a bifurcation point will be occurred. Then, the calculation goes back to the initial state of this step, and an eigenvalue analysis of $\left[K_{\mathrm{t}}\right]$ is conducted to obtain the current possible instability modes. With the chosen possible instability mode $\{v\}$, a most unfavorable wind loading distribution can be estimated by

$$
\left\{F_{W}\right\}=\left\{\bar{F}_{W}\right\}+\left\{\varepsilon_{i}\right\} \sigma_{F_{W}}=\left\{\bar{F}_{W}\right\}+\left\{\varepsilon_{i}\right\} g \rho_{a} \bar{V}_{h} \sigma_{v} A_{i}
$$

where, $\left\{\varepsilon_{i}\right\}$ is the normalized vector of the product, $\left[K_{t}\right]\{v\} .\left\{\varepsilon_{i}\right\}$ can be taken as the most unfavorable distribution estimation of fluctuating component of wind loading. $g$ is a peak factor with a range of 2.5-5.0; $\sigma_{v}$ is the standard deviation of the reference wind speed.

In this method, the amplitude of fluctuating component is considered with a uniform peak factor, $g$, as usual. Since the fluctuating component has random characteristics, the possible instability mode was used as a most unfavorable estimation of its distribution. Therefore, a conservative estimation of the equivalent static wind load of fluctuating component considering the effects of wind load on structural stability can be estimated. On the other hand, this method can get a suitable reference response variable, i.e., the displacement at the instability node, for using the effective static wind load distribution estimation method. 


\subsubsection{The universal equivalent static wind load method (Universal ESWL)}

As wind-induced responses vary in time and space, the largest load effects for all structural members do not occur simultaneously. The universal ESWL method makes it possible to produce a universal wind load distribution that simultaneously reproduces largest load effects for all structural members. The universal ESWL is shown by a combination of eigenmodes calculated by POD analysis of fluctuating wind pressure. Applying singular value decomposition to a matrix of eigenmodes by the influence function optimizes the universal ESWL combination as [7]

$$
\{\hat{R}\}=\left[I_{R}\right][\Phi][C]=\left[R_{0}\right][C]
$$

where $\{\hat{R}\}$ is the largest load effects of all structural members; $\left[I_{R}\right]$ is an influence function matrix; $[\Phi]$ is the eigenmode of norm 1 obtained from a proper orthogonal decomposition analysis of the fluctuating wind pressure field; $\left[R_{0}\right]$ is the product of the influence function and the eigenmode; $[C]$ is the contribution factor of the eigenmode of the equivalent static wind load distribution, which can be obtained as a least-square approximation of Eq.(7).

The universal ESWL distribution has the following features [7]: (1) The universal ESWL reproduces these largest load effects simultaneously; (2) The universal ESWL distribution is shown by summation of several load distributions; (3) The universal ESWL can simultaneously reproduce multiple load effects, e.g. bending moment and shear force.

\subsection{Dynamic time-history analysis for wind-induced responses}

Based on the theory of the dynamic finite element (FEM) method, the linear, even nonlinear vibration equations for spatial structures subjected to wind loading can be expressed as follows:

$$
[M]\left\{\ddot{U}_{t}\right\}+[C]\left\{\dot{U}_{t}\right\}+[K]\left\{U_{t}\right\}=\left\{F_{W}(t)\right\}
$$

where, $[M]$ is the mass matrix; $[C]$ the damping matrix; $[K]$ is the structural nonlinear stiffness matrix; $\{U\},\{\dot{U}\}$ and $\{\ddot{U}\}$ is the displacement, the velocity and the acceleration vectors, respectively; $\left\{F_{\mathrm{W}}(t)\right\}$ is the external dynamic load vector.

For spatial structures, it is necessary to expand the measured wind pressure from the limited measurement points to all the structural nodes on the surface in order to conduct a static or dynamic analysis since the effect of load distribution is very important in many cases. For the mean and fluctuating wind pressure coefficient distributions, they can be expanded by numerical interpolation simply. However, it is not suitable to expanding the time-series data of wind pressure in this way. By using the first $\mathrm{M}$ orthonormal eigenvectors obtained by POD as a coordinate system and utilizing their orthogonality, expanding wind pressure data to each point on the whole surface can be carried out within an acceptable error [8], i.e., the expanded wind pressure field can be expressed as [9]

$$
p_{i}^{e}(t)=\sum_{m=1}^{M} a_{m}(t) \varphi_{m i}^{e}
$$




$$
a_{m}(t)=\sum_{k=1}^{N} p_{k}(t) \phi_{m k} \Delta s_{k} / \sum_{k=1}^{N} \phi_{m k}^{2} \Delta s_{k}
$$

where, $\left\{\phi_{m i}\right\}$ and $\left\{\phi_{m i}^{e}\right\}$ is the $m$-th original and expanded orthogonal eigenvector, respectively, $m=1,2, \ldots, M, \mathrm{i}=1,2, \ldots, n$ and $n$ is the node number of the analysis model; $a_{m}(t)$ is the $\mathrm{m}$-th principal coordinate; $p_{k}(t)$ is the measured wind pressure data at point $k$, $k=1,2, \ldots, N, N$ is the number of measuring points; $\Delta s_{k}$ is the representative area of point $k$.

A full linear or non-linear time-history analysis using the measured wind tunnel data is the most accurate method for analysis of strength, deformation and stability of spatial structures. However, because it is very time-consuming, it is suitable primarily for final analysis.

\section{Wind-induced envelope responses in wind-resistant design of spatial structures}

\subsection{Difficulty and inconveniency in wind-resistant design for Spatial Structures at present}

\subsubsection{Necessity of wind tunnel tests}

Generally, as we all know, there are four kinds of methods which can be used to get the wind pressure distribution of a structure at present, i.e., 1) Codes on wind loading; 2) Wind tunnel tests; 3) Numerical simulation using the Computational Fluent Dynamics (CFD) technique; 4) Site full-scale measurement. Due to the complexity of the shapes of the spatial structures, it is difficult to provide some representative wind load information for spatial structures and hence most codes on wind loading only present wind pressure for representative low-rise and high-rise buildings. Numerical simulation is a promising solution to the wind pressure for spatial structures, but some problems still exists especially in the turbulence model in numerical simulation. And, site measuring is not available for the proposed structures in design. Therefore, wind tunnel tests become the main approach to obtain the wind pressure distribution for spatial structures at present.

\subsubsection{Complexity of wind load combination}

On the other hand, wind pressure distributions obtained from wind tunnel tests generally have many cases considering the wind attack angles. Currently, the unfavorable cases are used in the load combination through time-history analysis with wind tunnel experiment results. However, it is difficult to gain the most unfavorable wind load cases. If each case is considered, the analysis will be time-consuming and heavy. Then, effective and simplified method is necessary in the wind-resistant design, especially for large-span spatial structures.

\subsubsection{Time-consuming in wind-resistant analysis}


At present, the given information in all load codes for structural design in many countries may be only enough to use the gust factor type method, although sometimes we still need to know the so-called wind-induced dynamic effect coefficients by wind tunnel investigation. In order to using the effective static wind load distribution estimation method or the universal equivalent static wind load method, we need to know the co-relationship of the wind pressure between each two structural nodes, while this information can only be obtained by wind tunnel investigation case by case. Even in the near future, it is not easy to put such information into load codes for large-span spatial structures due to strong individuality and complexity of the architectural shape. On the other hand, even for a certain wind load case, estimation for the effective static wind load distribution or the universal equivalent static wind load can be not conveniently calculated by a structural designer, most possibly needs to be conducted by a professional research group or the technical center of a corresponding design institute.

\subsection{Conception of wind-induced envelope responses}

Wind-induced Envelope Responses mean the maximum or minimum responses, including displacement of any nodes, internal forces (axial force, bending moment, torsional moment, or extreme stress, etc.) of any elements, reaction of any supports, etc., of the structures in question under the expected wind load action with any attack angle. It can be taken as all the maximum or minimum values for each response, respectively, in a collection of the maximum or minimum wind-induced response result obtained one by one from the effective static wind load distribution estimation method under different wind attack angle, or all the maximum or minimum values for each response, respectively, in a collection of the maximum or minimum wind-induced response results obtained group by group from the universal equivalent static wind load method under different wind attack angle, and it is of course can be directly obtained from the dynamic time-history analysis by extracting all the maximum or minimum values for each response, respectively, in a collection of the maximum or minimum wind-induced response results case by case of different wind attack angle, although all of the above calculations are really time-consuming and mostly necessary to be based on wind tunnel investigation.

For the current design method of structures is based on load combination, it is not convenient to use structural responses. Therefore, it is necessary to convert the maximum responses to maximum load distribution. According to the finite element method, the maximum load can be obtained with the maximum structural responses and the stiffness matrix. However, for each elements there are many collections of maximum responses. To obtain an exclusive maximum load, the most unfavorable collection of responses can be estimated through checking the element with each collection of the responses or taking the maximum responses of all structural responses, such as all internal forces, conservatively.

\subsection{Applications of wind-induced envelope responses on wind-resistant design of Spatial Structures}

From the structural designer's point of view, it will be much more convenient to use the Wind-induced Envelope Responses directly in load effect combination of all the loads and 
actions in structural design. Therefore, a framework can be conducted for applying windinduced envelope responses on wind-resistant design of spatial structures as follows:

(1) A wind tunnel investigation using multi-channel simultaneous pressure measurement technique is necessary to be finished with necessary attack angles in consideration;

(2) Dynamic time-history analysis for wind-induced responses for all the test cases under different attack angles can be carried out based on wind tunnel investigation;

(3) Wind-induced envelope responses can be statistically obtained from the results of dynamic time-history analysis for all the test cases;

(4) Maximum load distribution is obtained with the envelope responses and the stiffness matrix;

(5) Maximum load distribution is applied on load combination of all the loads and actions in structural design of spatial structures, finally.

\subsection{Discussion on the Universal ESWL method and the WER method}

The limitation of the GRF method, the ESWL method and the unfavorable ESWL method in the application of wind resistant of spatial structures have been stated above. Discussion on the Universal ESWL and the WER method will be given in detail in the following. To explain the two methods, a spherical shell and a cylinder shell are used based on wind tunnel tests [6].

Figure 1 gives the maximum responses including displacements, moments and axial forces obtained by the universal ESWL method. It can be found that the maximum responses reproduced by the universal method are not good compared to the results of time history analysis except the maximum displacements in y direction of cylinder shell. Actually, if two responses need contradictory wind load distribution, then one wind load distribution can not reproduce the two responses simultaneously. As shown in Figure 2, the displacements of point 1 and 2 in $y$ direction are compatible responses while the displacements of point 1 and 2 in $\mathrm{z}$ direction are contradictive responses. As stated in Ref.7, the universal ESWL method is very effective for the wind loading estimation of cantilevered structures of which response obviously are compatible. However, for other types of spatial structures, it is not always effective. 


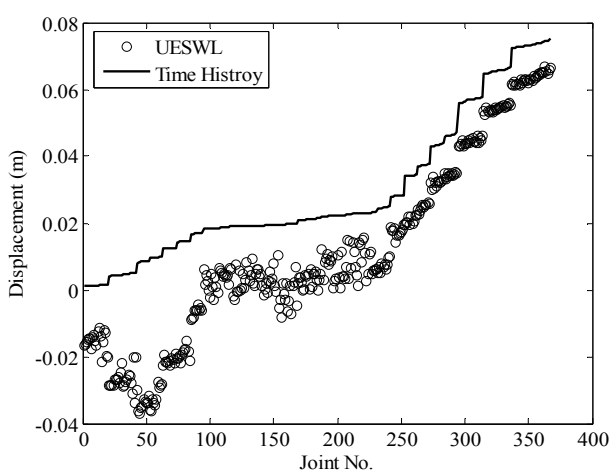

(a) Maximum displacements in $\mathrm{z}$ direction

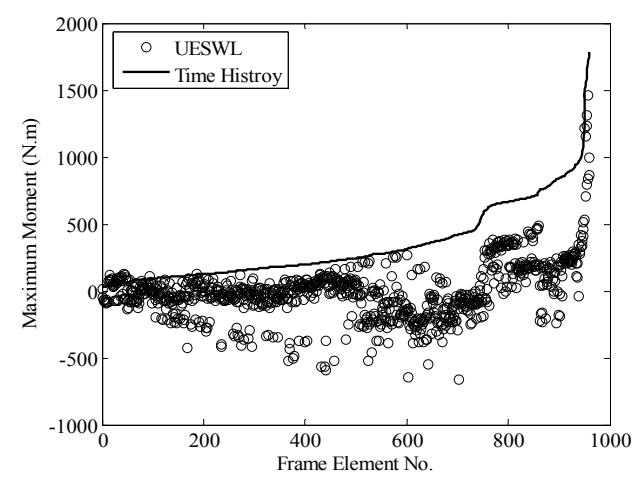

(c) Maximum moment

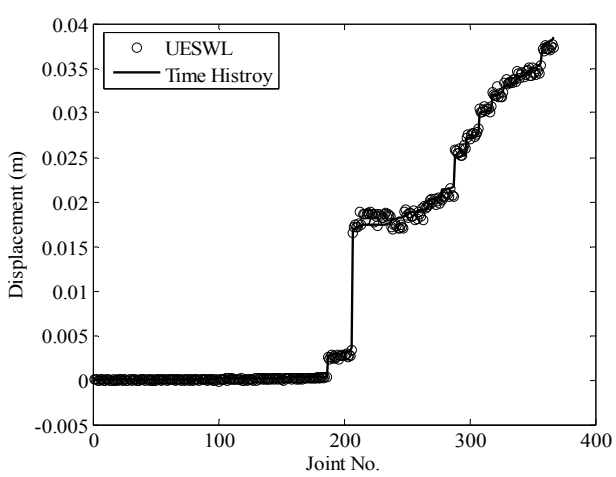

(b) Maximum displacements in y direction

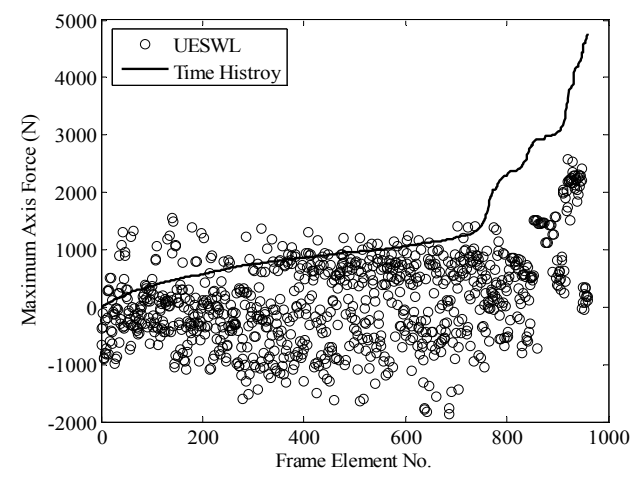

(d) Maximum axis forces

Figure 1: Maximum responses obtained with the universal ESWL for Cylindrical shell

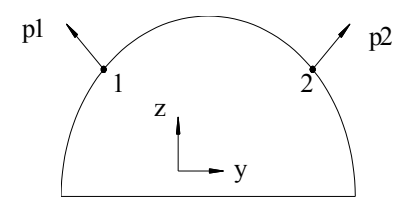

Figure 2: Compatible responses and contradictive responses

In addition, it seems that there is no improvement to use POD analysis in the estimation of effective static wind load, for the wind load reproducing maximum responses simultaneously does not real occur and has little relationship with the POD mode of the fluctuating wind pressure. 
To make the universal ESWL effective in general spatial structures, two methods can be used. One is to reduce the number of responses. Eq. (7) shows that if the number of responses are equal or less than the number of wind load measuring points, the exact wind load distribution can be obtained to reproduce the maximum responses simultaneously. But due to the limitation of measuring points, the number of responses is limited. Another is to modify the universal ESWL by dividing the responses into several groups. To get the same type of responses, the compatible coefficient $\alpha$ is proposed, as can be estimated by

$$
\alpha_{i k}=\sum_{j=1}^{N} I_{r i j} I_{r k j} / \sum_{j=1}^{N}\left|I_{r i j} I_{r k j}\right|
$$

where $\alpha_{i k}$ is the compatible coefficient of load effect $i$ and load effect $k ; I_{r i j}$ is the influence line of point $j$ on load effect $i$; $I_{r k j}$ is the influence line of point $k$ on load effect $j ; N$ is the number of wind load measuring points. If $\alpha=1$, the two responses are totally compatible, which means that they can be obtained from one wind load distribution. If $\alpha=0$, the two responses are totally contradictive to each other. If $\alpha=-1$, the two responses are totally negative compatible and also contradictive to each other. Obviously, the compatible coefficient $\alpha_{i k}$ represents the compatible degree of two responses. According to $\alpha$, the maximum responses can be divided into several groups as the following procedure:

1) Ascertain the critical value of the compatible coefficient $\alpha_{c r}$;

2) Choose an important responses $i$;

3) Calculate the compatible coefficients $\alpha_{i k}$ of response $i$ and the other response $k$, if the $\alpha_{i k}>\alpha_{c r}$, set the response $k$ into first group or else set into the next group;

4) Repeat the above steps for the leaved responses until all are set into groups.

Figure 3 gives the maximum axial forces obtained from the modified universal ESWL method. The modified universal ESWL could give more accurate results by setting the maximum responses into more groups. It can be found that the critical compatible coefficient can be chosen as a bigger value at the expense of more groups to get more exact results.

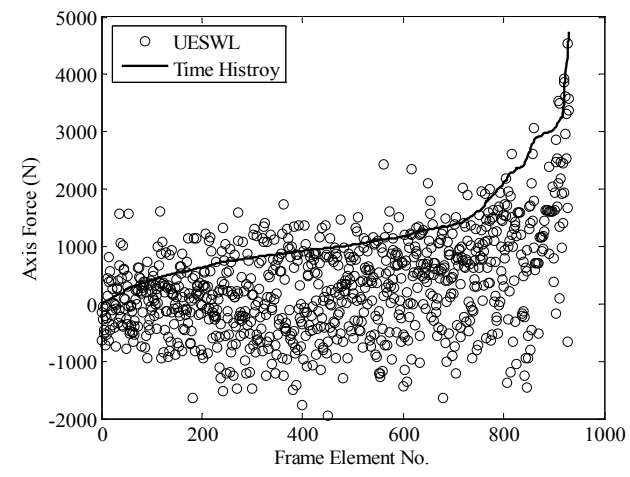

(a) $\alpha=-0.5$ (first group of 4 groups);

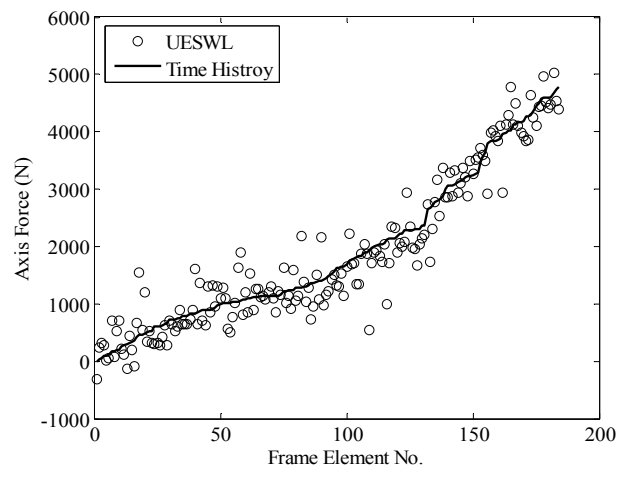

(b) $\alpha=0.5$ (first group of 28 groups)

Figure 3: Maximum axial forces for cylinder shell obtained with modified Universal ESWL 


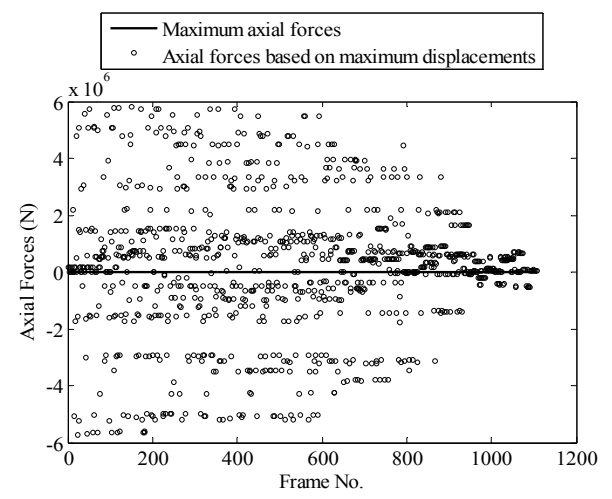

Figure 4: Comparison of the envelope axial forces and the axial forces obtained from envelope displacements

Obviously, the WER method could give exact maximum displacements responses. However, internal forces can not be obtained simultaneously. Figure 4 give the comparison of the maximum axial forces and the axial forces reproduced by the load distribution obtained with the stiffness matrix and the maximum displacement responses. To obtain the internal forces, element stiffness matrix could be used to obtain the displacements corresponding to maximum internal forces and then estimate the load distribution. Hence, two load distribution corresponding to envelope displacements and internal forces can be used for effective wind load estimation in design practice.

\subsection{Comparison}

In Table 1, comparison among the wind-resistant methods for spatial structures mentioned above is shown. From the table it can be found that, different methods have both advantages and disadvantages, while for wind-resistant design based on wind-induced envelope responses, it may be very convenient for large-span spatial structures, since wind tunnel test highly may necessary for such a project, and deep investigation for windinduced effect under so many different cases are always necessary in its structural design.

Table 1 Comparison among the wind-resistant methods for spatial structures

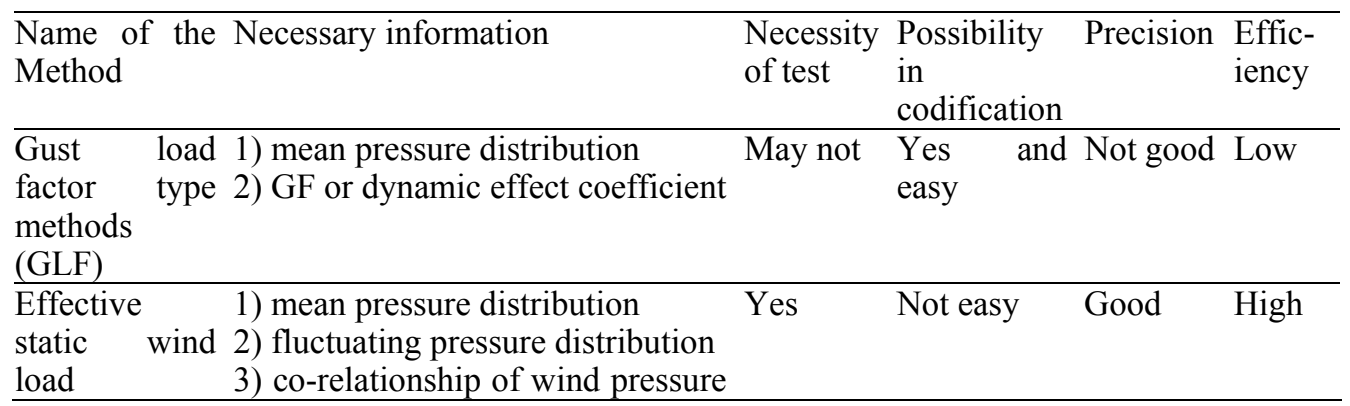




\begin{tabular}{|c|c|c|c|c|}
\hline $\begin{array}{l}\text { distribution } \\
\text { estimation } \\
\text { (ESWL) }\end{array}$ & $\begin{array}{l}\text { distribution } \\
\text { 4) peak factors } \\
\text { 5) structural influence matrix }\end{array}$ & & & \\
\hline $\begin{array}{l}\text { Unfavorable } \\
\text { ESWL }\end{array}$ & $\begin{array}{l}\text { 1) mean pressure distribution } \\
\text { 2) GF or dynamic effect coefficient } \\
\text { 3) peak factors }\end{array}$ & $\begin{array}{l}\text { Yes and } \\
\text { easy }\end{array}$ & $\begin{array}{l}\text { Conserva } \\
\text {-tive }\end{array}$ & Middle \\
\hline $\begin{array}{l}\text { Universal } \\
\text { ESWL }\end{array}$ & $\begin{array}{l}\text { 1) mean pressure distribution } \\
\text { 2) fluctuating pressure distribution possibly } \\
\text { 3) co-relationship of wind pressure may not } \\
\text { distribution } \\
\text { 4) peak factors } \\
\text { 5) structural influence matrix }\end{array}$ & Not easy & Best & Middle \\
\hline $\begin{array}{l}\text { Wind-induced } \\
\text { envelope } \\
\text { responses } \\
\text { (WER) }\end{array}$ & $\begin{array}{l}\text { 1) simultaneous time-history wind Yes } \\
\text { pressure data } \\
\text { 2) structural stiffness, mass and } \\
\text { damping matrix }\end{array}$ & No & Best & Middle \\
\hline
\end{tabular}

\section{Conclusions}

In this paper, the framework of Wind-induced Envelope Responses approach using for wind-resistant design of spatial structures is proposed from the structural designer's point of view. Advantages and disadvantages of the presented approach, as well as other windresistant methods for spatial structures were discussed and compared. Two numerical examples of spherical shell and cylinder shell were used to illuminate the efficiency of the presented approach. However, the present approach are mainly based on time-history analysis for wind-induced vibration under each wind attack angle, and the response combination of all loads and action will be used in structural design.

\section{Acknowledgement}

The authors are grateful to the financial support by the National Natural Science Foundation of China (No.50508024) and the 21st Century COE Program of Tokyo Polytechnic University awarded by the Ministry of Education, Culture, Sports, Science and Technology, Japan. In addition, this work is partly supported by the Visiting Scholar Fund of Key Laboratory of Concrete and Prestressed Concrete Structures (Southeast University), Ministry of Education, P.R.C.

\section{References}

[1] V. Gioncu, Buckling of Reticulated Shells: State-of-the-Art, Int. J. Space Structures, 10 (1995) 1-46.

[2] GB5009 (2001), China Loading Code for Buildings, China Plan Press. (In Chinese)

[3] Architectural Institute of Japan (AIJ), Recommendations for Loads on Buildings, Published by AIJ, Architectural Institute of Japan, 1996. (In Japanese) 
[4] G. Solari, Generalized Definition of Gust Factor, J. Wind Eng. Ind. Aerodyn., 36 (1990) 539-548.

[5] J. D. Holmes, Wind Loading of Structures, Spon Press, 2001.

[6] Y.Q. Li, Y. Tamura. A. Yoshida, A. Katsumura and K. Cho. Wind Loading and Its Effects on Single-Layer Reticulated Cylindrical Shells, J. Wind Eng. Ind. Aerodyn., 2006, 94(12): 949-973.

[7] A. Katsumura, Y. Tamura, O. Nakamura, Universal wind load distribution simultaneously reproducing largest load effects in all subject members on large-span cantilevered roof, J. Wind Eng. Ind. Aerodyn., 95 (2007) 1145-1165.

[8] Y. Tamura, S. Suganuma, H. Kikuchi and K. Hibi, Proper Orthogonal Decomposition of Random Wind Pressure Field, Journal of Fluids and Structures, 13 (1999) 10691095.

[9] Y.Q. Li and Y. Tamura, Wind-Resistant Analysis for Large-span Single-layer Reticulated Shells. Int. J. Space Structures, 19 (2004) 47-59. 